\title{
Coxsackievirus B3 protease 3C induces cell death in eukaryotic cells
}

\author{
Bannazadeh-Baghi H. ${ }^{1}$, Bamdad T. ${ }^{1 *}$, Soleimanjahi H. ${ }^{1}$, Kermanian M. ${ }^{2}$ \\ 1. Department of Virology, School of Medical Sciences, Tarbiat Modares University, \\ Tehran, Iran. \\ 2. Shahid Madany Hospital of Karaj, Iran University of Medical Sciences, Iran.
}

\begin{abstract}
Coxsackievirus B3 (CVB3) is the most common agent known to cause viral myocarditis. The viral genome encodes a single polyprotein that is cleaved to produce several proteins by virally encoded proteases. Most of this proteolytic processing is catalyzed by a cysteine protease called 3C. The 3C protease plays major role in viral replication and cellular damage. To understand the mechanism of $3 \mathrm{C}$ function in virus infected cells and also development of antiviral agants against the virus, a 3C expressing plasmid was constructed. The cDNA of 3C protease was synthesized using CVB3 infected cells through reverse transcription process and was cloned in pcDNA3.1-. The constructed plasmid was confirmed by sequencing and restriction enzyme analysis. By transfection of the constructed plasmid into HeLa and MCF-7 cells, we showed that 3C protease induced cell death through multiple converging pathways, such as down regulation of cellular factors and decreasing of mRNA transcripts. This affect on HeLa cells as stronger than MCF-7 cells.
\end{abstract}

Keywords: Protease $3 \mathrm{C} \bullet$ Cloning $\bullet$ Cell death $\bullet$ Coxsackievirus B3

\section{Introduction}

$(1$ oxsackievirus B3 (CVB3) is a positive single-stranded RNA virus in the genus Enterovirus of the family Picornaviridae. It is one of the six serotypes of coxsackievirus B group and its genome contains a single open reading frame flanked by the $5^{\prime}$ and $3^{\prime}$ untranslated regions (UTRs). The two virally encoded proteases, $2 \mathrm{~A}$ and $3 \mathrm{C}$, are the key proteins that control the virus replication cycle $(12,18)$. The structure of catalytic site of $3 \mathrm{C}$ protease is similar to the serine proteinase chymotrypsin; however, 3C protease uses a cysteine instead of serine as the nucleophilic amino acid in the catalytic triad $(15,17)$.

Protease 3C mediates cleavage of TATA-box binding protein (TBP), a transcription factor, which in turn prevents RNA polymerase II-mediated cellular transcription, whereas TBP cleavage by $2 \mathrm{~A}$ was unable to inhibit the function of $\operatorname{TBP}(7,24)$.

\footnotetext{
*Corresponding author: Bamdad T., Department of Virology, School of Medical Sciences, Tarbiat Modares University Tehran, Iran.

Tel.: +9821 82884525; Fax: +982188013030.

Email: Bamdad_T@modares.ac.ir
}

Protease 3C can also cleave ribosome-associated poly (A)-binding protein (RABP), removing its Cterminal domain that is important for interactions with other translation factors (13). Like protease 2A, 3C protease cleaves cellular proteins important in host gene expression. Therefore, these enzymes are attractive therapeutic targets for development of antiviral therapeutic agents (14).

Several picornaviruses, including poliovirus and coxsackievirus, are able to kill cells by apoptosis in a variety of situations $(4,5,23)$. This process may provide an efficient way to help virus spread to neighboring cells, to protect progeny viruses from host immune defenses, and to avoid an inflammatory response $(3,22)$. Virus-induced apoptosis could play a key role in the cytotoxicity associated with several human diseases of viral origin $(2,20)$.

The mentioned past studies on $3 \mathrm{C}$ protease function mainly focused on poliovirus (PV). The recent researches, demonstrated that $3 \mathrm{C}$ protease of CVB3 induced apoptosis through multiple converging pathways, such as induced cell death through cleavage or down regulation of cellular factors for translation and transcription (6). 
Coxsackievirus B3 protease $3 \mathrm{C}$ induces cell death in eukaryotic cells...

To more evaluate the $3 \mathrm{C}$ function and also to make an in vitro system for studying the effects of antiproteolitic agents on picornaviruses in the present study, we cloned the CVB3 cDNA segment encompassing the $3 \mathrm{C}$ coding region and then HeLa and MCF-7 cells were transfected with the constructed plasmid. After transfection, we showed the cells morphological changes by phase contrast microscopy and measured mRNA transcripts by Semi-quantitative RT-PCR.

\section{Materials and methods}

\section{Cells and virus}

HeLa and MCF-7 cells were cultured in DMEM (Dubelcco Minimal Eagle Medium)(Gibco,UK.) supplemented with $10 \%$ fetal bovine serum(FBS) (Gibco), 0.2\% sodium bicarbonate (sigma) and $100 \mathrm{IU} / \mathrm{ml}$ penicillin. HeLa cell line was used for CVB3 propagation (Nancy strain, American type tissue culture number VR-30).

\section{Bacterial strains and plasmids}

Escherichia coli DH5 $\alpha$ was used as a host during the cloning experiments and utilized for propagation of the plasmids. The expression vector pcDNA3.1 (Invitrogen, USA), was used for cloning and expression of truncated $\mathrm{CVB} 3 \mathrm{3C}$ protease gene.

\section{Viral RNA preparation}

HeLa cells at $80 \%$ confluency were infected with CVB3 at moi of $10 \mathrm{PFU}$ in a medium containing $1 \%$ FBS. Infected cells were collected after $24 \mathrm{~h}$ by centrifugation at $300 \times \mathrm{g}$ for $10 \mathrm{~min}$. Total RNA was extracted by the guanidine isothiocyanate based RNX-plus solution (SinnaGen INC., IRI) according to the manufacturer protocol as described elsewhere (11).

\section{DNA recombinant technology}

All molecular techniques including PCR and cloning were performed according to the standard methods (21). Briefly, after RNA extraction the CVB3 3C cDNA was synthesized by RT-PCR, using random hexamers. The flowing primers were used for amplification of 3C cDNA: 3Cf (5'CAAGCAAAATGCAAGG-3') and 3Cr (5'CTCTCAATAAATTATATTTCACC-3'). The BamHI and XbaI sites and start and stop codons were included at the 5' ends of forward and reverse primers, respectively. After digestion with BamHI and XbaI, the PCR product was inserted into the BamHI and XbaI sites of the vector pcDNA3.1under the control of CMV promoter. The resulted construct was verified by colony PCR, restriction enzyme analysis and DNA sequencing ( SEQLAB, Germany).

\section{Cell transfection}

HeLa and MCF-7 cells grown in 6-well plates were transfected with the plasmid (pcDNA3C) using Lipofectamin2000 (Invitrogen,USA). 3C-less plasmids-transfected cells were used as negative controls. The transfected cells were examined for morphological changes at 24,48 and $72 \mathrm{~h}$ post transfection.

Multiplex RT-PCR amplification and Semiquantitative analysis

Total RNA was isolated from cells $36 \mathrm{~h}$ after transfection using RNX-plus solution. $\quad \beta 2$ microglubolin mRNA was used as an indicator for total mRNAs isolates.

To compare the amount of $\beta 2$ microglubolin mRNA with a known concentration of RNA in semi-quantitative RT-PCR test, $1 \mu \mathrm{g}$ of entro virus RNA was added to each sample before RNA extraction. PCR amplification was performed with two sets of specific primers in one tube included the sense entrovirus ( $5^{\prime} \mathrm{ctg}$ at gcg gct aat cc $\left.3^{\prime}\right)$, anti sense entrovirus ( $5^{\prime}$ tgt cac cat aag cag cca $3^{\prime}$ ), sense b2m (5' tct ggg ttt cat cca tcc 3'), anti sense b2m (5' tac ctg tgg agc aac ctg 3'). PCR products were run on $2 \%$ agarose gel and photographed on top of a 280nm UV light box after ethidium bromid staining. The gel images were digitally captured with a CCD camera and analyzed with the National Institutes of Health (NIH) Imager beta version 2 programs. $\beta 2$ microglubolin mRNA content of each sample was measured semi quantitatively by band densitometry ratio of $\beta 2$ microglubolin to entrovirus bands.

\section{Results}

\section{PCR of 3C gene}

Coxsakievirus B3 was propagated in HeLa cells and the virus was harvested after $24 \mathrm{~h}$ while maximum cytopathic effect was observed. The titer of harvested virus was determined to be $10^{-5}$ TCID50/ml when calculated through SpearmanKärber formula.

PCR was performed using designed specific primers to obtain the $3 \mathrm{C}$ gene and the product of PCR (a 545 bp band) was evaluated through electrophoresis on $0.8 \%$ agarose gel (Fig. 1). 


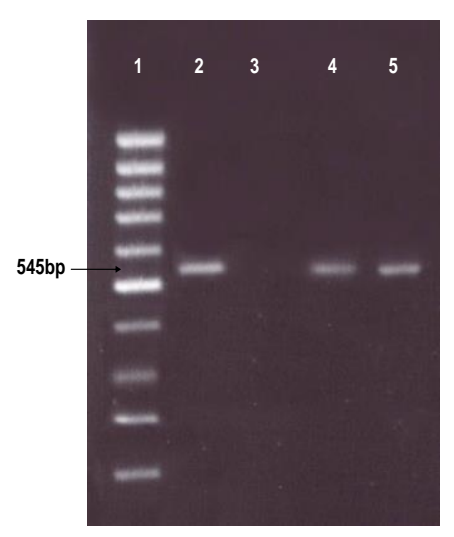

Fig. 1: Gel electrophoresis of PCR product of $3 C$ amplification.

Cloning of coxsackievirus B3 cDNA encoding 3C protease plasmid construction was done with reference to the CVB3 restriction map (Fig. 2) derived from the nucleotide sequence reported by Lindberg et al (16), and constructed plasmid was checked by colony PCR and restriction enzyme map analysis.

DNA sequence of the viral cDNA in pcDNA3.1was verified by sequencing (SEQLAB, Germany).

Morphological changes induced in cells expressing CVB3 3C protease to determine whether CVB3 3C protease expression affects cell viability, HeLa and MCF-7 cells were transfected with the constructed plasmid and cell morphological changes were observed by inverted microscope (Fig. 3). The

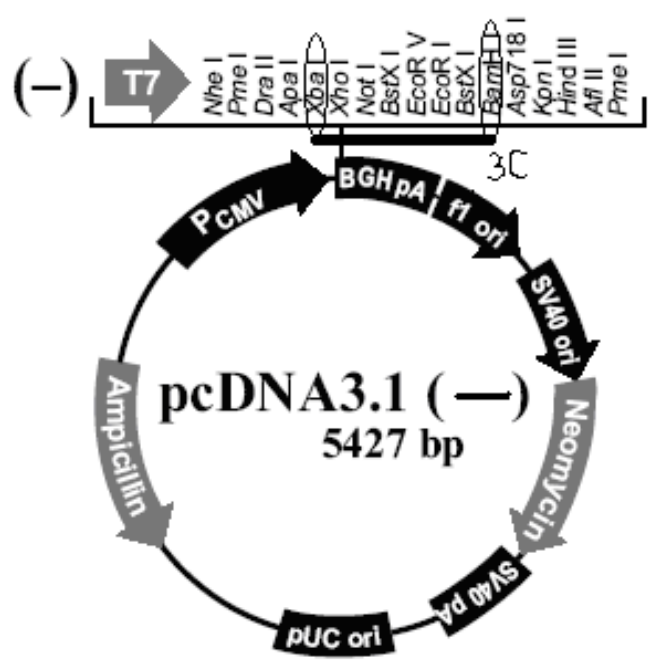

Fig. 2: Diagrams of the plasmid pcDNA.1- which used for the insertion of desired $3 \mathrm{C}$ gene.

appearance of cell shrinkage and loss of cell adherence began after $24 \mathrm{~h}$ for HeLa cells and $36 \mathrm{~h}$ for MCF-7 cells. After 96h most cells had died, as evidenced by the detached and rounded cells floating in the culture media. Cell death after post transfection of HeLa cells were more than MCF-7 cells.

HeLa and MCF-7 cells transfected with CVB3 3C protease gene were observed by invert microscope at the indicated time point post transfectsd. 3C-less plasmid transfected and untransfected cells were used as control.

\section{Semi-quantitative analysis}

Decreasing of mRNA was measured semiquantitatively by the band densitometry ratio of
3C-less plasmid

HeLa cells

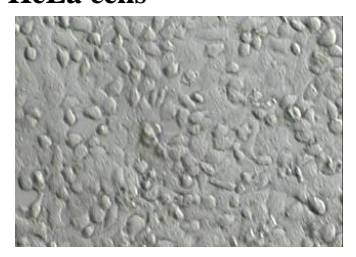

MCF-7 cells

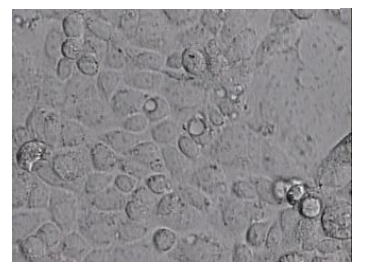

24h pt
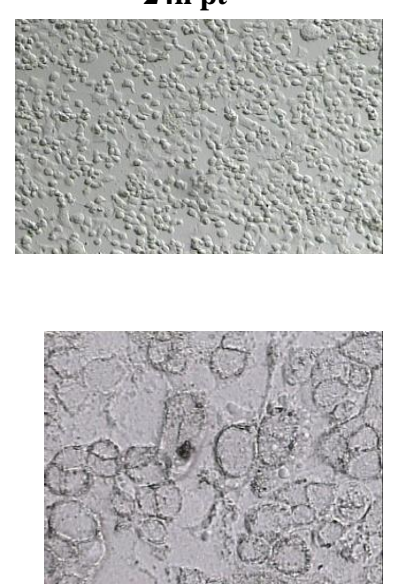

$96 h$ pt
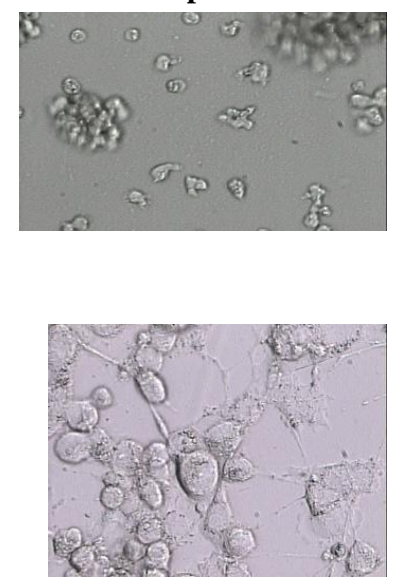

nontransfected
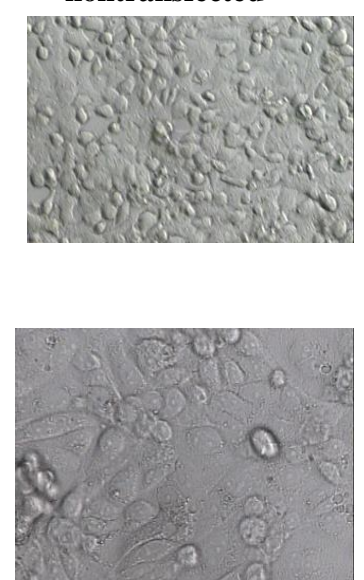

Fig. 3: Expression of CVB3 3C protease induced HeLa and MCF-7 cell death. 


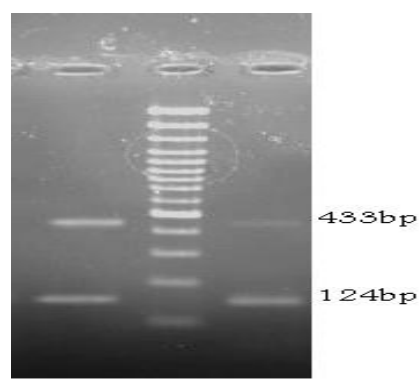

Fig. 4: Multiplex PCR was performed with entrovirus and $\beta 2 \mathrm{~m}$ set primers in one tube. The size of entrovirus and $\beta 2 \mathrm{~m}$ PCR product are 433 and $124 \mathrm{bp}$, respectively.

$\beta 2 \mathrm{~m}$ to entrovirus (Fig. 4). Values of $\mathrm{P} \leq 0.05$ were taken as significant (Fig. 5). The decrease of mRNA content for both cells was almost equal.

\section{Discussion}

Coxsackievirus B3 is an important human pathogen that has been associated with severe diseases. Clinically, coxsackievirus infections are known to be associated with acute and chronic viral myocarditis as well as pancreatitis $(1,18)$. It is well demonstrated that the picornavirus proteases $2 \mathrm{~A}$ and $3 \mathrm{C}$ cleave cellular proteins involving in translation like the translation initiation factor eIF4G (9), and the poly (A) binding protein (10), leading to a host translation shut-off. Two major and distinct modes of eukaryotic cell death are known: apoptosis and necrosis (8). Apoptosis is a common cellular response to a variety of viral infections which promotes virus release from apoptotic host cells results in the spread of virus particles (19). Recent study showed that the 3C protease of CVB3 induces apoptosis through multiple converging pathways (6).

In the present study the $3 \mathrm{C}$ gene of CVB3 was used to study the proteolytic effect of $3 \mathrm{C}$ which is conserved among all picornaviruses. The cDNA from CVB3 3C protease was prepared and cloned into eukaryotic expression vector, and then the constructed plasmid was transfected into HeLa and MCF-7 cells. Regarding the fast growing nature of these cells, HeLa and MCF-7 were chosen for better monitoring of cell changes. Investigation of the effect of the plasmid on the cell lines showed that cell death begun after $24 \mathrm{~h}$ post transfection. We showed this effect by cell morphological changes and semi-quantitative RT-PCR. These results suggest that although $3 \mathrm{C}$ changes protein content of cells $(7,13)$, it may affect mRNAs level

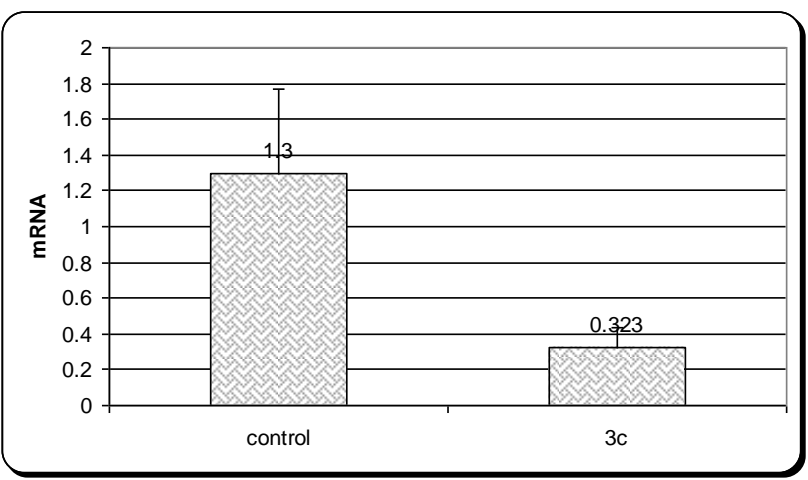

Fig. 5: The $\beta 2 m$ mRNA level in transfected cells. The results showed that mRNA level in transfected cells (3c) has significantly decreased comparing to non-transfected cells (control).

by probable degradation of proteins involving in mRNA processing.

The results of this study, suggest that $3 \mathrm{C}$ protease is thought to be a suitable target for antiviral chemotherapy.

\section{References}

1. Baboonian, C., M. J. Davies, J. C. Booth, and W. J. McKenna. 1997. Coxsackie B viruses and human heart disease. Curr Top Microbiol Immunol 223:31-52.

2. Barco, A., E. Feduchi, and L. Carrasco. 2000. Poliovirus protease $3 \mathrm{C}$ (pro) kills cells by apoptosis. Virology 266:352-360.

3. Brien, V. 1998. Viruses and apoptosis. J. Gen. Virol. 79:1833-1845.

4. Carthy, C. M., D. J. Granville, K. A. Watson, D. R. Anderson, J. E. Wilson, D. Yang, D. W. Hunt, and B. M. McManus. 1998. Caspase activation and specific cleavage of substrates after coxsackievirus B3-induced cytopathic effect in HeLa cells. J Virol 72:7669-7675.

5. Castelli, J., B. A. Hassel, K. A. Wood, X. L. Li , K. Amemiya, M. C. Dalakas, P. F. Torrence, and R. J. Youle. 1997. A study of the interferon cytometry intransiently transfected cells. Nucleic Acids Res. 25:4855-4857.

6. Chau, D. H., J. Yuan, H. Zhang, P. Cheung, T. Lim, Z. Liu, A. Sall, and D. Yang. 2007. Coxsackievirus B3 proteases 2A and $3 \mathrm{C}$ induce apoptotic cell death through mitochondrial injury and cleavage of eIF4GI but not DAP5/p97/NAT1. Apoptosis 12:513-524. 
7. Clark, M. E., P. M. Lieberman, A. J. Berk, and A. Dasgupta. 1993. Direct cleavage of human TATA-binding protein by poliovirus protease $3 \mathrm{C}$ in vivo and in vitro. Mol Cell Biol 13:12321237.

8. Ellis, R. E., J. Y. Yuan, and H. R. Horvitz. 1991. Mechanisms and functions of cell death. Annu Rev Cell Biol 7:663-698.

9. Haghighat, A., Y. Svitkin, I. Novoa, E. Kuechler, T. Skern, and N. Sonenberg. 1996. The eIF4G-eIF4E complex is the target for direct cleavage by the rhinovirus $2 \mathrm{~A}$ proteinase. J Virol 70:8444-8450.

10. Kerekatte, V., B. D. Keiper, C. Badorff, A. Cai, K. U. Knowlton, and R. E. Rhoads. 1999. Cleavage of Poly(A)-binding protein by coxsackievirus $2 \mathrm{~A}$ protease in vitro and in vivo: another mechanism for host protein synthesis shutoff? J Virol 73:709-717.

11. Kermanian, M., H. Soleimanjahi, E. Arefian, and T. Bamdad. 2008. Enrichment of cerebrospinal fluid samples on cell culture for enhancement of sensitivity of mumps and enterovirus detection by multiplex RT-PCR. Diagn Microbiol Infect Dis 60:375-379.

12. Klump, W. M., I. Bergmann, B. C. Muller, D. Ameis, and R. Kandolf. 1990. Complete nucleotide sequence of infectious Coxsackievirus B3 cDNA: two initial 5' uridine residues are regained during plus-strand RNA synthesis. J Virol 64:1573-1583.

13. Kuyumcu-Martinez, N. M., M. Joachims, and R. E. Lloyd. 2002. Efficient cleavage of ribosome-associated poly(A)-binding protein by enterovirus 3C protease. J Virol 76:2062-74.

14. Lall, M. S., R. P. Jain, and J. C. Vederas. 2004. Inhibitors of $3 \mathrm{C}$ cysteine proteinases from Picornaviridae. Curr Top Med Chem 4:12391253.

15. Lawson, M. A., and B. L. Semler. 1991. Poliovirus thiol proteinase $3 \mathrm{C}$ can utilize a serine nucleophile within the putative catalytic triad. Proc Natl Acad Sci U S A 88:9919-23.

16. Lindberg, A. M., P. O. Stalhandske, and U. Pettersson. 1987. Genome of coxsackievirus B3. Virology 156:50-63.

17. Mosimann, S. C., M. M. Cherney, S. Sia, S. Plotch, and M. N. James. 1997. Refined X-ray crystallographic structure of the poliovirus 3C gene product. J Mol Biol 273:1032-1047.

18. Pallansch, M., and R. Roos. Fields; 2007; Enteroviruses: polioviruses, Coxsackieviruses, echoviruses, and newer enteroviruses, p. 839894. In D. M. Knipe, P. A. Philadelphia, K. W., and L. W. (ed.), Fields Virology, Lippincott Raven, Philadelphia.

19. Roulston, A., R. C. Marcellus, and P. E. Branton. 1999. Viruses and apoptosis. Annu Rev Microbiol 53:577-628.

20. Rudin, C. M., and C. B. Thompson. 1997. Apoptosis and disease: regulation and clinical relevance of programmed cell death. Annu Rev Med 48:267-281.

21. Sambrook, J., and D. W. Russell 2001. Molecular cloning: A Laboratory Manual, Third edition,. Cold Spring Harbor Laboratory Press.

22. Teodoro, J. G., and P. E. Branton. 1997. Regulation of apoptosis by viral gene products. J Virol 71:1739-1746.

23. Tolskaya, E. A., L. I. Romanova, M. S. Kolesnikova, T. A. Ivannikova, E. A. Smirnova, N. T. Raikhlin, and V. I. Agol. 1995. Apoptosis-inducing and apoptosispreventing functions of poliovirus. J Virol 69:1181-1189.

24. Yalamanchili, P., R. Banerjee, and A. Dasgupta. 1997. Poliovirus-encoded protease 2APro cleaves the TATA-binding protein but does not inhibit host cell RNA polymerase II transcription in vitro. J Virol 71:6881-6886. 